\title{
A NOTE ON PRÜFER SEMISTAR MULTIPLICATION DOMAINS
}

\author{
Giampaolo Picozza
}

\begin{abstract}
In this note we give a new generalization of the notions of Prüfer domain and $\mathrm{P} v \mathrm{MD}$ which uses quasi semistar invertibility, the "quasi $\mathrm{P} \star \mathrm{MD}$ ", and compare them with the $\mathrm{P} \star \mathrm{MD}$. We show in particular that the problem of when a quasi $\mathrm{P} \star \mathrm{MD}$ is a $\mathrm{P} \star \mathrm{MD}$ is strictly related to the problem of the descent to subrings of the $\mathrm{P} \star \mathrm{MD}$ property and we give necessary and sufficient conditions.
\end{abstract}

\section{Introduction}

In [12], Houston, Malik and Mott defined the notion of $\star$-multiplication domain, in order to generalize the notions of Prüfer domain and $\mathrm{P} v \mathrm{MD}$ to arbitrary star operations. After the introduction of semistar operations by Okabe and Matsuda [14], Fontana, Jara and Santos [5] have defined Prüfer semistar multiplication domains ( $\mathrm{P} \star \mathrm{MD}$ 's), as a further generalization to the semistar context of these concepts. To define $\mathrm{P} \star \mathrm{MD}$ 's they used a notion of $\star$-invertibility analogous to the one already used for star operations. In [7] we have introduced the notion of quasi semistar invertibility, which is more natural in the semistar context. In this note we want to give a new generalization of the notions of Prüfer domain and $\mathrm{P} v \mathrm{MD}$ which uses quasi semistar invertibility, the "quasi $\mathrm{P} \star \mathrm{MD}$ ", and compare them with the $\mathrm{P} \star \mathrm{MD}$. We show in particular that the problem of when a quasi $\mathrm{P} \star \mathrm{MD}$ is a $\mathrm{P} \star \mathrm{MD}$ is strictly related to the problem of the descent to subrings of the $\mathrm{P} \star \mathrm{MD}$ property and we give necessary and sufficient conditions. An interesting case is when $\star$ is the $b$-operation (which associates to each ideal its integral closure). In fact, it turns out that QPbMD's are exactly the domains with Prüfer integral closure.

At the end of the paper, we briefly address the problem of giving a similar generalization of the notion of semistar Dedekind domain introduced in [2] (the quasi $\star$-Dedekind domain) but we show that some of the good properties of semistar Dedekind domains are not preserved. Both in the case of QP $\star$ MD's

Received January 24, 2008.

2000 Mathematics Subject Classification. 13A15.

Key words and phrases. semistar operation, star operation. 
and quasi- $\star$-Dedekind domains we show that when $\star=\tilde{\star}$ we obtain the equivalence with $\mathrm{P} \star \mathrm{MD}$ 's and $\star$-Dedekind domains, and these are the cases in which things work well. So, even if the notions of quasi Prüfer semistar multiplication domain and quasi semistar Dedekind domain are probably more natural in the semistar context it turns out that the notions of $\mathrm{P} \star \mathrm{MD}$ and $\star$-Dedekind domain are the ones which better generalize the classical case.

Let $D$ be an integral domain. We denote by $\overline{\boldsymbol{F}}(D)$ the set of $D$-submodules of the quotient field $K$ of $D$, by $\boldsymbol{F}(D)$ the set of nonzero fractional ideals of $D$ and by $\boldsymbol{f}(D)$ the set of nonzero finitely generated fractional ideals of $D$.

A semistar operation on $D$ is a map $\star: \bar{F}(D) \rightarrow \overline{\boldsymbol{F}}(D), E \mapsto E^{\star}$, such that, for all $x \in K, x \neq 0$, and for all $E, F \in \overline{\boldsymbol{F}}(D)$, the following properties hold:

$\left(\star_{1}\right)(x E)^{\star}=x E^{\star}$

$\left(\star_{2}\right) E \subseteq F$ implies $E^{\star} \subseteq F^{\star}$;

$\left(\star_{3}\right) E \subseteq E^{\star}$ and $E^{\star \star}:=\left(E^{\star}\right)^{\star}=E^{\star}$.

A (semi)star operation $\star$ on an integral domain $D$ is a semistar operation that restricted to the set of fractional ideals is a star operation. It is easy to see that a semistar operation $\star$ is a (semi)star operation if and only if $D^{\star}=D$.

If $\star$ is a semistar operation on $D$, then we can consider a map $\star_{f}: \overline{\boldsymbol{F}}(D) \rightarrow$ $\overline{\boldsymbol{F}}(D)$ defined for each $E \in \overline{\boldsymbol{F}}(D)$ as follows: $E^{\star_{f}}:=\bigcup\left\{F^{\star} \mid F \in \boldsymbol{f}(D)\right.$ and $F \subseteq$ $E\}$. It is easy to see that $\star_{f}$ is a semistar operation on $D$, called the semistar operation of finite type associated to $\star$. Note that, for each $F \in \boldsymbol{f}(D), F^{\star}=$ $F^{\star}{ }_{f}$. A semistar operation $\star$ is called a semistar operation of finite type if $\star=\star_{f}$. It is easy to see that $\left(\star_{f}\right)_{f}=\star_{f}$ (that is, $\star_{f}$ is of finite type).

As usual, we denote by $v$ (or $v_{D}$ ) the (semi)star operation on $D$ defined by $E^{v}=(D:(D: E))$ for all $E \in \bar{F}(D)$ and by $t$ (or $t_{D}$ ) the (semi)star operation of finite type associated to $v$. If $T$ is an overring of $D$, the map $E \mapsto E^{\star_{T}}=E T$ for all $E \in \overline{\boldsymbol{F}}(D)$, is a semistar operation of finite type, which is not a (semi)star operation when $T \supsetneq D$.

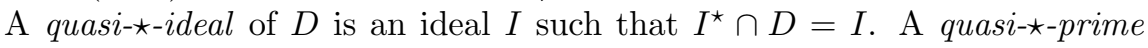
is a prime ideal that is also a quasi- $\star$-ideal. A quasi- $\star-$ maximal ideal is a (proper) ideal that is maximal in the set of all quasi- $\star$-ideals of $D$. If $\star$ is a semistar operation of finite type, each quasi- - -ideal of $D$ is contained in a quasi-^-maximal ideal. Moreover, each quasi- $\star$-maximal ideal of $D$ is prime [4, Lemma 4.20].

If $\star_{1}$ and $\star_{2}$ are two semistar operations on $D$, we say that $\star_{1} \leq \star_{2}$ if $E^{\star_{1}} \subseteq E^{\star_{2}}$ for each $E \in \overline{\boldsymbol{F}}(D)$.

We say that a semistar operation is stable if $(E \cap F)^{\star}=E^{\star} \cap F^{\star}$ for each $E, F \in \overline{\boldsymbol{F}}(D)$.

If $\star$ is a semistar operation on $D$, we denote by $\approx$ the semistar operation $\star_{\mathcal{M}\left(\star_{f}\right)}$ induced by the set $\mathcal{M}\left(\star_{f}\right)$ of the quasi- $-\star_{f}$-maximal ideals of $D$, that is, $E^{\tilde{\star}}=\bigcap_{P \in \mathcal{M}\left(\star_{f}\right)} E D_{P}$ for each $E \in \overline{\boldsymbol{F}}(D)$. The semistar operation $\tilde{\star}$ is stable 
and of finite type and $\tilde{\star} \leq \star_{f}$. The operation $\tilde{v}$ is usually denoted by $w$, since it coincides with the $w$-operation studied in [19].

A semistar operation $\star$ is a.b. (resp. e.a.b.), if for all $E \in \boldsymbol{f}(D)$ and $F, G \in$ $\boldsymbol{F}(D)$ (resp. $F, G \in \boldsymbol{f}(D)),(E F)^{\star} \subseteq(E G)^{\star}$ implies $F^{\star} \subseteq G^{\star}$.

The Kronecker functions ring $\operatorname{Kr}(D, \star)$ of an integral domain $D$ with respect to a semistar operation $\star$ is the ring

$$
\begin{aligned}
\operatorname{Kr}(D, \star):= & \{f / g \mid f, g \in D[X] \backslash\{0\} \text { there exists } h \in D[X] \backslash\{0\} \\
& \text { such that } \left.(\boldsymbol{c}(f) \boldsymbol{c}(h))^{\star} \subseteq(\boldsymbol{c}(g) \boldsymbol{c}(h))^{\star}\right\} \cup\{0\} .
\end{aligned}
$$

(We denote by $\boldsymbol{c}(h)$ the content of a polynomial $h$.)

The Nagata ring $\mathrm{Na}(D, \star)$ of $D$ with respect to $\star$ is the ring

$$
\mathrm{Na}(D, \star):=\left\{\frac{f}{g}, f, g \in D[X], g \neq 0,(c(g))^{\star}=D^{\star}\right\} .
$$

For more results on semistar operations and in particular on Nagata rings and Kronecker function rings, see for example [6].

Finally, recall that if $\star$ is a semistar operation on $D, T$ an overring of $D$ and $\iota$ the canonical embedding of $D$ in $T$, then $\star$ induces a semistar operation $\star_{\iota}$ by restriction to $\overline{\boldsymbol{F}}(T)$, that is, $E^{\star_{\iota}}=E^{\star}$ for all $E \in \overline{\boldsymbol{F}}(T)$. Conversely, if $*$ is a semistar operation on $T$, we can define a semistar operation $*^{\iota}$ on $D$, setting $E^{*^{\iota}}=(E T)^{*}$ for all $E \in \overline{\boldsymbol{F}}(D)$ (For more results on these operations, see [15]).

\section{2. $\mathrm{QP} \star \mathrm{MD}$ 's and the descent of the $\mathrm{P} \star \mathrm{MD}$ property}

As we mentioned in the introduction, in [5], M. Fontana, P. Jara, and E. Santos have introduced the notion of a $\mathrm{P} \star \mathrm{MD}$ to generalize the concepts of Prüfer domains, PvMD's and $\star$-multiplication domains to the context of semistar operations.

Let $D$ be an integral domain and $\star$ a semistar operation on $D$. We say that $D$ is a Prüfer $\star-$ multiplication domain $(\mathrm{P} \star \mathrm{MD}$ for short) if each finitely generated nonzero ideal $I$ of $D$ is $\star_{f}$-invertible (recall that an ideal $I$ is $\star$ invertible if $\left.\left(I I^{-1}\right)^{\star}=D^{\star}\right)$.

Since $\star_{f}$-invertibility and $\approx$-invertibility coincide [7, Proposition 2.18], we have that $D$ is a $\mathrm{P} \star \mathrm{MD}$ if and only each finitely generated nonzero ideal of $D$ is $\tilde{\star}$-invertible.

Clearly $D$ is a $\mathrm{P} \star \mathrm{MD}$ if and only if $D$ is a $\mathrm{P} \star_{f} \mathrm{MD}$, if and only if $D$ is a $\mathrm{P} \tilde{\star} \mathrm{MD}$.

Let $\star_{1} \leq \star_{2}$ be two semistar operations on $D$. It is easy to see that, if $D$ is a $\mathrm{P} \star_{1} \mathrm{MD}$, then $D$ is a $\mathrm{P} \star_{2} \mathrm{MD}$.

The following characterization of $\mathrm{P} \star \mathrm{MD}$ 's is due to M. Fontana, P. Jara, and E. Santos [5, Theorem 3.1, Remark 3.1] and generalizes several known results about PvMD's (cf. M. Griffin [10, Theorem 5], R. Gilmer [8, Theorem 2.5], J. Arnold and J. Brewer [1, Theorem 3], J. Querré [18, Théorème 3, p. 279], B. G. Kang [13, Theorem 3.5, Theorem 3.7] and M.Zafrullah [20, Theorem 11]). 
Proposition 2.1. Let $D$ be an integral domain and $\star$ a semistar operation on $D$. The following are equivalent:

(i) $D$ is a $P \star M D$.

(ii) $D_{Q}$ is a valuation domain for each $Q \in \mathcal{M}\left(\star_{f}\right)$.

(iii) $\mathrm{Na}(D, \star)$ is a Prüfer domain.

(iv) $\mathrm{Na}(D, \star)=\operatorname{Kr}(D, \star)$.

(v) $\tilde{\star}$ is an e.a.b. semistar operation.

(vi) $\star_{f}$ is stable and e.a.b.

In particular, in a $P \star M D, \tilde{\star}=\star_{f}$.

In [7], we have introduced the notion of quasi- $\star$-invertibility, as a generalization, "typical" of the semistar context, of the notion of $\star$-invertibility: an ideal $I$ of $D$ is quasi- $\star$-invertible if there exists $H \in \overline{\boldsymbol{F}}(D)$ such that $(I H)^{\star}=D^{\star}$. This is equivalent to saying that $I^{\star}$ is $\star_{\iota}$-invertible in $D^{\star}$ (where $\iota$ is the canonical embedding of $D$ in $\left.D^{\star}\right)$. It is clear that a $\star$-invertible ideal is quasi- $\star$-invertible, but not all quasi- $\star$-invertible ideals are $\star$-invertible ([7, Example 2.9]).

We add three other equivalent conditions to Proposition 2.1, relating the notion of $\mathrm{P} \star \mathrm{MD}$ with quasi- $\star$-invertibility.

Proposition 2.2. Let $D$ be an integral domain and $\star$ a semistar operation on D. The following are equivalent:

(i) $D$ is a $P \star M D$.

(ii) Each finitely generated nonzero ideal of $D$ is quasi- - -invertible.

(iii) $D^{\star}=D^{\tilde{\star}}$ and each finitely generated nonzero ideal of $D$ is quasi $-\star_{f}-$ invertible.

(iv) $\star_{f}$ is stable and a.b.

Proof. (i) $\Leftrightarrow$ (ii) We have noticed that $D$ is a $\mathrm{P} \star \mathrm{MD}$ if and only if each finitely generated nonzero ideal of $D$ is $\tilde{\star}$-invertible. By [7, Corollary $2.17(2)]$, we deduce immediately that, since $\tilde{\star}$ is a stable semistar operation, $\tilde{\star}$-invertibility and quasi $-\tilde{\star}$-invertibility coincide for finitely generated ideals. Hence the assertion is proved.

(i) $\Rightarrow$ (iii) By definition of $\mathrm{P} \star \mathrm{MD}$, each finitely generated nonzero ideal of $D$ is $\star_{f}$-invertible and then quasi $-\star_{f}$-invertible. Moreover, $\star_{f}=\tilde{\star}$ (Proposition 2.1). Then, $D^{\tilde{\star}}=D^{\star} f=D^{\star}$.

(iii) $\Rightarrow$ (ii) Since $D^{\star}=D^{\tilde{\star}}$, a quasi- $\star_{f}$-invertible ideal is also quasi- $\tilde{\star}$-invertible ([7, Proposition 2.21]).

(i) $\Rightarrow$ (iv) We know already (Proposition $2.1(\mathrm{i}) \Rightarrow($ vi) $)$ that $\star_{f}$ is stable. Let $E \in \boldsymbol{f}(D), \quad F, G \in \overline{\boldsymbol{F}}(D)$, such that $(E F)^{\star_{f}}=(E G)^{\star_{f}}$. From the definition of $\mathrm{P} \star \mathrm{MD}, \quad\left(E E^{-1}\right)^{\star_{f}}=D^{\star}$. We have that $\left(E^{-1}(E F)^{\star_{f}}\right)^{\star_{f}}=\left(E^{-1}(E G)^{\star_{f}}\right)^{\star_{f}}$. Thus, $F^{\star_{f}}=\left(\left(E^{-1} E\right)^{\star_{f}} F\right)^{\star_{f}}=\left(\left(E^{-1} E\right)^{\star_{f}} G\right)^{\star_{f}}=G^{\star_{f}}$.

(iv) $\Rightarrow$ (i) It is a straightforward consequence of Proposition 2.1(vi) $\Rightarrow$ (i) since an a.b. semistar operation is e.a.b. 
The next example shows that the requirement that $D^{\star}=D^{\tilde{}}$ cannot be removed from condition (iii).

Example 2.3. Let $D$ be a pseudovaluation non valuation domain, that is, a local domain with maximal ideal $M$, such that $M^{-1}=V$ is a valuation domain with maximal ideal $M([11])$. Let $\star:=\star_{\{V\}}\left(\right.$ note that $\left.\star=\star_{f}\right)$. Since $M$ is the only quasi- $\star_{f}$-maximal, $\tilde{\star}=d$, so in particular $\tilde{\star} \neq \star_{f}$ and $D$ is not a $\mathrm{P} \star \mathrm{MD}$. But it is easy to see that each finitely generated nonzero ideal $I$ of $D$ is quasi- $-\star_{f}$-invertible (since $I V$ is invertible in $V$ ). Note that in this case $D=D^{\tilde{\star}} \neq V=D^{\star}$.

So, a domain in which each finitely generated nonzero ideal is quasi- $\star_{f}{ }^{-}$ invertible is not in general a $\mathrm{P} \star \mathrm{MD}$. It is natural to investigate the structure of the integral domains having this property, as a generalization of $\mathrm{P} \star \mathrm{MD}$ 's.

Let $D$ be an integral domain and $\star$ a semistar operation on $D$. We say that $D$ is a $Q P \star M D$ (quasi Prüfer semistar multiplication domain) if each finitely generated nonzero ideal of $D$ is quasi- $\star_{f}$-invertible.

Since $a \star_{f}$-invertible ideal is quasi- $\star_{f}$-invertible, a $\mathrm{P} \star \mathrm{MD}$ is always a $\mathrm{QP} \star \mathrm{MD}$, but the converse is not always true, as Example 2.3 shows.

As we have already noticed, the notions of $\mathrm{P} \star \mathrm{MD}, \mathrm{P} \star_{f} \mathrm{MD}$ and $\mathrm{P} \approx \mathrm{MD}$ coincide. Clearly $D$ is a $\mathrm{QP} \star \mathrm{MD}$ if and only if $D$ is a $\mathrm{QP} \star_{f} \mathrm{MD}$, while there exist $\mathrm{QP} \star \mathrm{MD}$ 's that are not $\mathrm{QP} \approx \mathrm{MD}$. In fact, from Proposition 2.2 it follows that a domain $D$ is a $\mathrm{QP} \approx$ MD if and only if $D$ is a $\mathrm{P} \star \mathrm{MD}$. So, in particular, Example 2.3 gives an example of a $\mathrm{QP} \star \mathrm{MD}$ which is not a $\mathrm{QP} \approx \mathrm{MD}$.

It is easy to see that if $\star_{1} \leq \star_{2}$ are two semistar operations on $D$ and $D$ is a $\mathrm{QP} \star_{1} \mathrm{MD}$, then $D$ is also a $\mathrm{QP} \star_{2} \mathrm{MD}$, so, in particular, a $\mathrm{QP} \tilde{\star} \mathrm{MD}$ is always a $\mathrm{QP} \star \mathrm{MD}$.

Finally, we remark that for a (semi)star operation $\star$, the notions of $\mathrm{P} \star \mathrm{MD}$ and $\mathrm{QP} \star \mathrm{MD}$ coincide, since in this case $D^{\star}=D$ and so the notions of $\star-$ invertibility and quasi- $\star$-invertibility coincide ([7, Corollary 2.17]).

The next lemma relates the notions of $\mathrm{QP} \star \mathrm{MD}$ and of $\mathrm{P} v \mathrm{MD}$, showing that a $\mathrm{QP} \star \mathrm{MD}$ is in fact a particular subring of a $\mathrm{P} v \mathrm{MD}$. This implies that the study of a $\mathrm{QP} \star \mathrm{MD}$ can be done studying a particular overring which is a $\mathrm{P} v \mathrm{MD}$.

Lemma 2.4. Let $D$ be an integral domain and $\star$ a semistar operation on $D$. Let $\iota$ be the canonical inclusion of $D$ in $D^{\star}$. The following are equivalent:

(i) $D$ is a $Q P \star M D$.

(ii) $D^{\star}$ is a $P \star_{\iota} M D$.

(iii) $D^{\star}$ is a PvMD and $\star_{f}=t\left(D^{\star}\right)\left(:=\left(t_{D^{\star}}\right)^{\iota}\right)$, the descent to $D$ of the t-operation of $D^{\star}$

Proof. (i) $\Rightarrow$ (ii) Let $I=\left(a_{1}, a_{2}, \ldots, a_{n}\right) D^{\star}$ be a finitely generated ideal of $D^{\star}$. Consider $I_{0}=\left(a_{1}, a_{2}, \ldots, a_{n}\right) D$. Since $D$ is a $\mathrm{QP} \star \mathrm{MD}, I_{0}$ is quasi$\star_{f}$-invertible. So, $I_{0}^{\star}$ is $\left(\star_{f}\right)_{\iota}$-invertible, that is, $\left(\star_{\iota}\right)_{f}$-invertible (since in this case $\left(\star_{f}\right)_{\iota}=\left(\star_{\iota}\right)_{f}\left[16\right.$, Proposition 2.8]). But $\left(I_{0}\right)^{\star}=\left(\left(a_{1}, a_{2}, \ldots, a_{n}\right) D\right)^{\star}=$ 
$\left(\left(a_{1}, a_{2}, \ldots, a_{n}\right) D^{\star}\right)^{\star}=I^{\star}$. Thus, $I^{\star}$ (and consequently $\left.I\right)$ is $\left(\star_{\iota}\right)_{f}$-invertible. Hence $D^{\star}$ is a $\mathrm{P} \star_{\iota} \mathrm{MD}$.

(ii) $\Rightarrow$ (iii) Since $\star_{\iota}$ is a (semi)star operation on $D^{\star}$, we have that $D^{\star}$ is a $\mathrm{P} v \mathrm{MD}$ and $\left(\star_{f}\right)_{\iota}=\left(\star_{\iota}\right)_{f}=t_{D^{\star}}$, the $t$-operation of $D^{\star}([5$, Proposition 3.4]). Thus, $\star_{f}=\left(\left(\star_{f}\right)_{\iota}\right)^{\iota}=\left(t_{D^{\star}}\right)^{\iota}=t\left(D^{\star}\right)$.

(iii) $\Rightarrow\left(\right.$ i) Let $I$ be a finitely generated nonzero ideal of $D$. Since $\left(\star_{f}\right)_{\iota}=$ $\left(t\left(D^{\star}\right)\right)_{\iota}=t_{D^{\star}}$, we have $\left(I\left(D^{\star}: I\right)\right)^{\star}=\left(I D^{\star}\left(D^{\star}: I D^{\star}\right)\right)^{\star}=\left(\left(I D^{\star}\right)^{t_{D^{\star}}}\left(D^{\star}\right.\right.$ : $I))^{t_{D^{\star}}}=D^{\star}$, since $D^{\star}$ is a $\mathrm{P} v \mathrm{MD}$.

So, we can restate the analogue of Proposition 2.1 for QP $\star$ MD's:

Proposition 2.5. Let $D$ be an integral domain and $\star$ a semistar operation on $D$. Let $\iota$ be the canonical embedding of $D$ in $D^{\star}$. The following are equivalent:

(i) $D$ is a $Q P \star M D$.

(ii) $\left(D^{\star}\right)_{Q}$ is a valuation domain for each $Q \in \mathcal{M}\left(\left(\star_{f}\right)_{\iota}\right)$.

(iii) $\mathrm{Na}\left(D^{\star}, \star_{\iota}\right)$ is a Prüfer domain.

(iv) $\operatorname{Na}\left(D^{\star}, \star_{\iota}\right)=\operatorname{Kr}\left(D^{\star}, \star_{\iota}\right)(=\operatorname{Kr}(D, \star))$.

$(\mathrm{v})(\tilde{\star})_{\iota}$ is an e.a.b. semistar operation.

(vi) $\star_{f}$ is e.a.b. and $\left(\star_{f}\right)_{\iota}$ is stable.

In stating condition (iv) we have used the fact that $\operatorname{Kr}(D, \star)=\operatorname{Kr}\left(D^{\star}, \star_{\iota}\right)$. For condition (vi) we have used that $\star_{f}$ is e.a.b. if and only if $\left(\star_{f}\right)$, is e.a.b. (it is a straightforward consequence of Propositions 3.1, 3.2 and 3.5 of [15]).

Example 2.6. The operation which associates to each $E \in \overline{\boldsymbol{F}}(D)$ its integral closure is denoted by $b$ (i.e., $E^{b}=\bigcap E V$, where $V$ varies over the set of all valuation overrings of $D$ ). A domain $D$ with Prüfer integral closure is always a QPbMD. Indeed, it is easy to see that, if $\iota$ is the canonical embedding of $D$ in $D^{b}$, the integral closure of $D$, then $b_{\iota}$ is the $b$-operation of $D^{b}$, which is the identity, since $D^{b}$ is a Prüfer domain $([9$, Theorem 24.7$])$. So, in particular, $D^{b}$ is a $\mathrm{P} b_{\iota} \mathrm{MD}$ and $D$ is a $\mathrm{QP} b \mathrm{MD}$, by Lemma 2.4 .

Note that if $D$ is not itself a Prüfer domain, $D$ is not a PbMD. Indeed, $\tilde{b}=d$ ([17, Corollary 2.16]), and so $D$ is a PbMD if and only if $D$ is a Prüfer domain.

Conversely, if $D$ is a $\mathrm{QP} b \mathrm{MD}$, then $D^{b}$ is a $\mathrm{P} b_{\iota} \mathrm{MD}$ (Lemma 2.4), where, as we have noticed, $b_{\iota}$ is the $b$-operation of $D^{b}$. So, again by [17, Corollary 2.16], $D^{b}$, the integral closure of $D$, is a Prüfer domain.

So we have shown that:

$A$ domain $D$ is a $Q P b M D$ if and only if $D$ has Prüfer integral closure.

(Note that in Gilmer's book [9, Section 32] the notation $D^{b}$ is used to indicate the Kronecker function ring with respect to the $b$-operation, that is, the ring $\operatorname{Kr}(D, b)$. For us, $D^{b}$ is simply the $b$-closure of $D$, that is, its integral closure.)

Remark 2.7. Note that in the situation of Example 2.6 the $b$-operation of $D$ is the extension to the integral closure of $D$. In fact, we have the following result:

$A$ domain $D$ has Prüfer integral closure if and only if the b-operation of $D$ coincides with the extension to the integral closure of $D$. 
If $D$ has Prüfer integral closure $D^{\prime}$, as we have noticed, $b_{\iota}$ is the identity, and so, since $b=\left(b_{\iota}\right)^{\iota}\left(\left[15\right.\right.$, Proposition 3.5]), $b=\star_{D^{\prime}}$. Conversely, if $b=\star_{D^{\prime}}$, then $b_{\iota}=d_{D^{\prime}}$, the identity operation of $D^{\prime}$. But we have already noticed that $b_{\iota}=b_{D^{\prime}}$ is the $b$-operation of $D^{\prime}$. Thus $b_{D^{\prime}}=d_{D^{\prime}}$ and $D^{\prime}$ is a Prüfer domain by [9, Theorem 24.7].

The next corollary gives a way to construct all the QP $\star$ MD's:

Corollary 2.8. Let $T$ be a PvMD and let $D$ be a subring of $T$. Let $\star:=t(T)$, the descent to $D$ of the $t$-operation of $T$. Then, $D$ is a $Q P \star M D$. (Note that, by Lemma 2.4, all the $Q P \star M D$ 's can be constructed in this way.)

Proof. It is similar to Lemma 2.4 (iii) $\Rightarrow$ (i).

Remark 2.9. By Proposition 2.5, the semistar operation $\tilde{\star}$ on $D$ is a.b. if and only if $D$ is a $\mathrm{QP} \approx \mathrm{MD}$ (that is, a $\mathrm{P} \star \mathrm{MD}$ ). This is not true for an arbitrary semistar operation of finite type, that is, if $\star_{f}$ is a.b., $D$ is not necessarily a $\mathrm{QP} \star \mathrm{MD}$. For example, if $D$ is a $v$-domain which is not a $\mathrm{P} v \mathrm{MD}$ (recall that a $v$-domain is a domain in which the $v$-operation is e.a.b., or equivalently, a domain in which each finitely generated ideal is $v$-invertible), we have that the $t$-operation is a.b. (it is easy to see that $t$ is e.a.b. if and only if $v$ is e.a.b. and $t$ is e.a.b. if and only if $t$ is a.b. [9, Exercise 2, p. 406]), but $D$ is not a QPtMD (since a $\mathrm{QP} t \mathrm{MD}$ is precisely a $\mathrm{P} v \mathrm{MD}$ ).

Moreover, if $T$ is a $v$-domain which is not a $\mathrm{P} v \mathrm{MD}$ and $D$ a subring of $T$, the semistar operation $t(T)$ on $D$ is a.b., since it is the descent of the $t$-operation of $T$ which is a.b., but clearly $D$ is not a $\mathrm{QP} t(T) \mathrm{MD}$, since $D^{t(T)}=T$ is not a $\mathrm{P} v \mathrm{MD}$.

In [5, Proposition 3.1], the authors show that the property of being a $\mathrm{P} \star \mathrm{MD}$ ascends to overrings, that is, if $T$ is an overring of a $\mathrm{P} \star \mathrm{MD} D$, then $T$ is a $\mathrm{P} \star_{\iota} \mathrm{MD}$ (where $\iota$ is the canonical embedding of $D$ in $T$ ). On the contrary, they show $([5$, Example 3.2$])$ that the $\mathrm{P} \star \mathrm{MD}$ property does not descend always to subrings.

We show that the property of being a $\mathrm{QP} \star \mathrm{MD}$ both ascends and descends.

Proposition 2.10. Let $D$ be an integral domain, $T$ an overring of $D$ and $\iota$ the canonical embedding of $D$ in $T$. Then

(1) If $\star$ is a semistar operation on $D$ such that $D$ is a $Q P \star M D$, then $T$ is a $Q P \star_{\iota} M D$.

(2) If * is a semistar operation on $T$ such that $T$ is a $Q P * M D$, then $D$ is a $Q P *^{\iota} M D$.

Proof. (1) Let $I$ be a finitely generated nonzero ideal of $T$. Then $I=\left(a_{1}, a_{2}\right.$, $\left.\ldots, a_{n}\right) T$ for some $a_{1}, a_{2}, \ldots, a_{n} \in T$. Let $I_{0}=\left(a_{1}, a_{2}, \ldots, a_{n}\right) D$, the fractional ideal of $D$ generated by the $a_{i}$ 's. Since $D$ is a $\mathrm{QP} \star \mathrm{MD}$, we have $\left(I_{0}\left(D^{\star}: I_{0}\right)\right)^{\star}=$ $D^{\star}$. Since $T^{\star} \supseteq D^{\star}$, we have $T^{\star}=\left(I_{0}\left(D^{\star}: I_{0}\right)\right)^{\star} T^{\star} \subseteq\left(\left(I_{0}\left(D^{\star}: I_{0}\right)\right)^{\star} T^{\star}\right)^{\star} \subseteq$ $\left(I_{0} T\left(T^{\star}: I_{0} T\right)\right)^{\star}=\left(I\left(T^{\star_{\iota}}: I\right)\right)^{\star_{\iota}}$ and so $I$ is quasi- $\star_{\iota}$-invertible. 
(2) Note that $D^{*^{\iota}}=T^{*}$. Let $I$ be a finitely generated nonzero ideal of $D$. Since $I T$ is a finitely generated ideal of $T$ and $T$ is a $\mathrm{QP} * \mathrm{MD}$, there exists $H \in \overline{\boldsymbol{F}}(T) \subseteq \overline{\boldsymbol{F}}(D)$ such that $(I T H)^{*}=T^{*}$ and so $(I H)^{*^{\iota}}=D^{*^{\iota}}$, that is, $I$ is quasi- $*^{\iota}$-invertible.

We note that as a consequence of Lemma 2.4 (i) $\Leftrightarrow($ ii), the problem of studying when a $\mathrm{QP} \star \mathrm{MD}$ is a $\mathrm{P} \star \mathrm{MD}$ is equivalent to the study of when the fact that $D^{\star}$ is a $\mathrm{P} \star_{\iota} \mathrm{MD}$ (where $\star$ is a semistar operation on $D$ and $\iota$ is the canonical embedding of $D$ in $D^{\star}$ ) implies that $D$ is a $\mathrm{P} \star \mathrm{MD}$.

So, the next goal is to study when a $\mathrm{QP} \star \mathrm{MD}$ is a $\mathrm{P} \star \mathrm{MD}$ and deduce results on the descent of the $\mathrm{P} \star \mathrm{MD}$ property.

We have already shown that when $\star=\tilde{\star}$ and when $\star$ is a (semi)star operation a $\mathrm{QP} \star \mathrm{MD}$ is always a $\mathrm{P} \star \mathrm{MD}$.

In [5, Proposition 3.2], the authors prove that the flatness of $T$ over $D$ is a sufficient condition for the $\mathrm{P} \star \mathrm{MD}$ property to descend from $T$ to $D$. So, analogously, the flatness of $D^{\star}$ over $D$ is sufficient for a $\mathrm{QP} \star \mathrm{MD} D$ to be a $\mathrm{P} \star \mathrm{MD}$.

We want to improve this result, giving necessary and sufficient conditions.

While $\operatorname{Kr}(D, \star)=\operatorname{Kr}\left(D^{\star}, \star_{\iota}\right)$ always, it is not always true that $\operatorname{Na}(D, \star)=$ $\mathrm{Na}\left(D^{\star}, \star_{\iota}\right)$ (this is true for example when $\star=\tilde{\star}$ ). It turns out that this condition on the Nagata ring is the key for the problem we are addressing, as the next proposition shows.

Proposition 2.11. Let $D$ be an integral domain, $\star$ a semistar operation on $D$ and $\iota$ the canonical embedding of $D$ in $D^{\star}$. The following are equivalent:

(i) $D$ is a $P \star M D$.

(ii) $D$ is a $Q P \star M D$ and $\mathrm{Na}(D, \star)=\mathrm{Na}\left(D^{\star}, \star_{\iota}\right)$.

Proof. It follows immediately from Proposition 2.5(iv) and Proposition 2.1(iv).

We show that the condition on the Nagata ring in (ii) of Proposition 2.11, is strictly related to the notion of semistar flatness, as studied in [3].

We start recalling some results on quasi- - -ideals and on semistar flatness.

Lemma 2.12. [6, Lemma 2.3(3)(4)] Let D be an integral domain, $\star$ a semistar operation on $D$ and $\iota$ the embedding of $D$ in $D^{\star}$.

(1) If $Q$ is a quasi $\star_{f}-$ maximal ideal of $D$, then there exists a quasi $-\left(\star_{f}\right)_{\iota}-$ maximal ideal $M$ of $D^{\star}$ such that $Q=M \cap D$.

(2) If $N$ is a quasi- $\left(\star_{f}\right)_{\iota}$-prime ideal of $D^{\star}$, then $N \cap D$ is a quasi $\star_{f}-$ prime ideal of $D$.

Let $D$ be an integral domain and $T$ an overring of $D$. Let $\star$ be a semistar operation on $D$ and let $\star^{\prime}$ be a semistar operation on $T$. We say [3, Section 3] that $T$ is $\left(\star, \star^{\prime}\right)$-linked to $D$ if, for each finitely generated nonzero integral ideal $F$ of $D$, we have that $F^{\star}=D^{\star}$ implies $(F T)^{\star^{\prime}}=T^{\star^{\prime}}$. We say [3, Section 4] 
that $T$ is $\left(\star, \star^{\prime}\right)$-flat over $D$ if $T$ is $\left(\star, \star^{\prime}\right)$-linked to $D$ and for each $Q \in \mathcal{M}\left(\star_{f}^{\prime}\right)$, $D_{Q \cap D}=T_{Q}$.

Proposition 2.13 ([3, Lemma 3.1(e), Proposition 3.2, Corollary 5.4, Theorems 4.5 and 5.7]). Let $D$ be an integral domain and $\star$ a semistar operation on $D$. Let $T$ be an overring of $D$ and $\star^{\prime}$ a semistar operation on $T$.

(1) $T$ is $\left(\star, \star_{\iota}\right)$-linked to $D$ (where $\iota$ is the canonical embedding of $D$ in $\left.T\right)$.

(2) $T$ is $\left(\star, \star^{\prime}\right)$-linked to $D$ if and only if $(N \cap D)^{\star} f \neq D^{\star}$ for each quasi$\star_{f}^{\prime}-$ maximal ideal $N$.

(3) $T$ is $\left(\star, \star^{\prime}\right)$-flat over $D$ if and only if $\mathrm{Na}\left(T, \star^{\prime}\right)$ is a flat overring of $\mathrm{Na}(D, \star)$.

(4) If $D$ is a $P \star M D$ and $T$ is $\left(\star, \star^{\prime}\right)$-linked to $D$, then $T$ is a $P \star M D$.

(5) $D$ is a $P \star M D$ if and only if for each overring $R$ of $D$ and for each semistar operation $\star^{\prime}$ on $R$ such that $R$ is $\left(\star, \star^{\prime}\right)$-linked to $D$ then $R$ is $\left(\star, \star^{\prime}\right)$-flat over $D$.

We can now show that our condition on the Nagata ring is in fact equivalent to the $\left(\star, \star_{\iota}\right)$-flatness of $D^{\star}$ over $D$.

Proposition 2.14. Let $D$ be an integral domain, $\star$ a semistar operation on $D$ and $\iota$ the canonical embedding of $D$ in $D^{\star}$. Then, the following are equivalent:

(i) $\mathrm{Na}(D, \star)=\mathrm{Na}\left(D^{\star}, \star_{\iota}\right)$.

(ii) $D^{\star}$ is $\left(\star, \star_{\iota}\right)$-flat over $D$.

(iii) $\left(D^{\star}\right)_{P}=D_{P \cap D}$ for each $P \in \mathcal{M}\left(\left(\star_{\iota}\right)_{f}\right)$.

(iv) $D^{\star}=D^{\tilde{\star}}$ and $\widetilde{\left(\star_{\iota}\right)}=(\tilde{\star})_{\iota}$.

Proof. (i) $\Rightarrow$ (ii) It follows immediately from Proposition 2.13(3).

(ii) $\Rightarrow$ (iii) It is trivial.

(iii) $\Rightarrow$ (iv) First we note that, in this case, $\left(\star_{f}\right)_{\iota}=\left(\star_{\iota}\right)_{f}[16$, Proposition 2.8]. Now, let $E \in \overline{\boldsymbol{F}}\left(D^{\star}\right)$. Then,

$$
E^{\tilde{\star}}=\bigcap_{Q \in \mathcal{M}\left(\star_{f}\right)} E D_{Q} \text { and } E^{\tilde{\star_{\iota}}}=\bigcap_{M \in \mathcal{M}\left(\left(\star_{f}\right)_{\iota}\right)} E\left(D^{\star}\right)_{M} .
$$

For each $Q \in \mathcal{M}\left(\star_{f}\right)$ there exists $M \in \mathcal{M}\left(\left(\star_{f}\right)_{\iota}\right)$ such that $Q=M \cap D$ (Lemma 2.12(1)). Since, by the hypothesis, $D_{Q}=\left(D^{\star}\right)_{M}$, we have that $\widetilde{E^{\left(\star_{\iota}\right)}} \subseteq E^{\tilde{\star}}$. Conversely, if $M$ is a quasi- $\left(\star_{f}\right)_{\iota}$-maximal ideal, then $M \cap D$ is a quasi- $\star_{f}$-prime ideal of $D$ (Lemma 2.12(2)). So, $M \cap D \subseteq Q$ for some $Q \in \mathcal{M}\left(\star_{f}\right)$. It follows that $D_{Q} \subseteq D_{M \cap D}=\left(D^{\star}\right)_{M}$. Thus $E^{\tilde{\star}} \subseteq E^{\left(\star_{\iota}\right)}$ and so $E^{\tilde{\star}}=\widetilde{E^{\left(\star_{\iota}\right)}}$. Moreover $D^{\star}=D^{\tilde{\star}}$. Indeed $D^{\star} \subseteq \bigcap_{M \in \mathcal{M}\left(\left(\star_{f}\right)_{\iota}\right)}\left(D^{\star}\right)_{M}=$ $\bigcap_{Q \in \mathcal{M}\left(\star_{f}\right)} D_{Q}=D^{\tilde{\star}} \subseteq D^{\star}$.

(iv) $\Rightarrow$ (i) By the hypothesis, $D^{\star}=D^{\tilde{\star}}$ and so, $\iota$ is also the canonical embedding of $D$ in $D^{\tilde{\star}}$. Thus we have $\mathrm{Na}(D, \star)=\mathrm{Na}(D, \tilde{\star})=\mathrm{Na}\left(D^{\tilde{\star}},(\tilde{\star})_{\iota}\right)=$ $\mathrm{Na}\left(D^{\star}, \widetilde{\left(\star_{\iota}\right)}\right)=\mathrm{Na}\left(D^{\star}, \star_{\iota}\right)$ (recalling that $\mathrm{Na}(D, \tilde{\star})=\mathrm{Na}\left(D^{\tilde{\star}}, \tilde{\star}_{\iota}\right)$ [6, Corollary 3.5$])$. 
Corollary 2.15. Let $D$ be an integral domain and $T$ an overring of $D$. Let $\iota$ be the canonical embedding of $D$ in $T$ and let $*$ be a semistar operation on $T$. Then, the following are equivalent:

(i) $\mathrm{Na}\left(D, *^{\iota}\right)=\mathrm{Na}(T, *)$.

(ii) $T$ is $\left(*^{\iota}, *\right)$-flat over $D$.

(iii) $T_{P}=D_{P \cap D}$ for each $P \in \mathcal{M}\left(*_{f}\right)$.

(iv) $T=\widetilde{*^{\iota}}$ and $\tilde{*}=\left(\widetilde{*^{\iota}}\right)_{\iota}$.

Proof. Apply Proposition 2.14 to $\star:=*^{\iota}$, recalling that $*=\left(*^{\iota}\right)_{\iota}$ (see for example [14, Lemma 45]).

We have the following result for $\mathrm{QP} \star \mathrm{MDs}$ and a corollary for the descent of the $\mathrm{P} \star \mathrm{MD}$ property.

Proposition 2.16. Let $D$ be an integral domain and $\star$ a semistar operation on D. Assume that $D$ is a $Q P \star M D$. Then, $D$ is a $P \star M D$ if and only if the equivalent conditions of Proposition 2.14 hold.

Proof. It is a consequence of Proposition 2.11.

Corollary 2.17. Let $D$ be an integral domain, $T$ an overring of $D$. Let $\iota$ be the canonical embedding of $D$ in $T$ and $*$ a semistar operation on $T$. Assume that $T$ is a $P * M D$. Then, $D$ is a $P *^{\iota} M D$ if and only if the equivalent conditions of Corollary 2.15 hold.

Proof. It follows from Proposition 2.16, since $T$ is a $\mathrm{P} * \mathrm{MD}$ if and only if $D$ is a $\mathrm{QP} *^{\iota} \mathrm{MD}$.

As a corollary we recover the results proven in [5, Proposition 3.2 and Proposition 3.3]. First we prove an easy lemma.

Lemma 2.18. Let $D$ be an integral domain, $\star$ a semistar operation on $D$ and $\iota$ the canonical embedding of $D$ in $D^{\star}$. Then $D^{\tilde{\star}}$ is $\left(\tilde{\star},(\tilde{\star})_{\iota}\right)$-flat over $D$.

Proof. It is immediate by Proposition 2.13, since $\mathrm{Na}(D, \star)=\mathrm{Na}\left(D^{\tilde{\star}},(\tilde{\star})_{\iota}\right)([6$, Corollary 3.5]).

Corollary 2.19 (cf. [5, Proposition 3.2 and Proposition 3.3]). Let $D$ be an integral domain.

(1) Let $T$ be a flat overring of $D$ and let $*$ be a semistar operation on $T$. Let $\iota:=\iota_{T}$ be the canonical embedding of $D$ in $T$. Assume that $T$ is a $P * M D$. Then $D$ is a $P *^{\iota} M D$.

(2) Let $\star$ be a semistar operation on D. Let $\iota$ be the canonical embedding of $D$ in $D^{\tilde{\star}}$. Then $D$ is a $P \star M D$ if and only if $D^{\tilde{\star}}$ is a $P(\tilde{\star})_{\iota} M D$.

Proof. (1) It is immediate by Corollary 2.17 .

(2) It is immediate by Lemma 2.18 and Proposition 2.16.

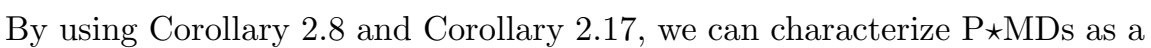
particular class of subrings of $\mathrm{P} v \mathrm{MDs}$. 
Theorem 2.20. Let $D$ be an integral domain and $\star$ a semistar operation on $D$. The following are equivalent:

(i) $D$ is a $P \star M D$;

(ii) There exists an overring $T$ of $D$ such that $T$ is a PvMD, $\star_{f}=t(T)$ and for each $t_{T}$-maximal ideal $Q$ of $T, T_{Q}=D_{Q \cap D}$.

(iii) There exists an overring $T$ of $D$ such that $T$ is a PvMD, $\star_{f}=t(T)$ and $T$ is $\left(t(T), t_{T}\right)$-flat over $D$.

Proof. (i) $\Rightarrow$ (ii) Let $T:=D^{\star}$. Thus $T$ is a $\mathrm{P} v \mathrm{MD}$ and $\star_{f}=t(T)$ (Lemma 2.4 $(\mathrm{i}) \Rightarrow($ iii $))$. Moreover, by Proposition $2.1 \star_{f}=\approx$. Thus, by Lemma $2.18 D^{\star}$ is $\left(\star_{f},\left(\star_{f}\right)_{\iota}\right.$ )-flat over $D$ (where $\iota$ is the canonical embedding of $D$ in $T$ ). Since $t_{T}=(t(T))_{\iota}=\left(\star_{f}\right)_{\iota}$, the assertion is proven.

(ii) $\Rightarrow$ (i) Recall that $D$ is a $\mathrm{P} \star \mathrm{MD}$ if and only if it is a $\mathrm{P} \star_{f} \mathrm{MD}$. Let $\iota$ be the canonical embedding of $D$ in $T$. Since $T$ is a $\mathrm{P} v_{T} \mathrm{MD}$, it is a $\mathrm{P} t_{T} \mathrm{MD}$. By the hypothesis on the $t_{T}$-maximal ideals, applying Corollary 2.17 , we have that $D$ is a $\mathrm{P}\left(t_{T}\right)^{\iota} \mathrm{MD}$, that is, a $\mathrm{P} \star_{f} \mathrm{MD}$ (recalling that, by definition, $\left.\left(t_{T}\right)^{\iota}=t(T)\right)$ ).

(ii) $\Leftrightarrow$ (iii) follows immediately by Corollary 2.15 (ii) $\Leftrightarrow$ (iii), since by definition $t(T)=\left(t_{T}\right)^{\iota}$.

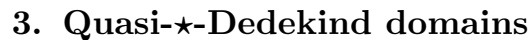

A domain $D$ with a semistar operation $\star$ is called $\star$-Noetherian if the ascending chain condition on quasi- $\star$-ideals holds (see [2] and [16]).

If one defines a $\star$-Dedekind domain $(\mathrm{a} \star$-DD) to be a $\star$-Noetherian $\mathrm{P} \star \mathrm{MD}$, one recovers semistar versions of all the main characterizations and properties of Dedekind domains, using the semistar operation $\tilde{\star}([2])$. So, $\star$-Dedekind domains are a very good generalization of Dedekind domains (and of Krull domains, since Krull domains are exactly the $v$-Dedekind domains [2, Remark $4.2(1)])$.

It is natural to ask wether the notion of $\mathrm{QP} \star \mathrm{MD}$ leads to a notion of "quasi$\star$-Dedekind domain", which preserves the same good characterizations for $\star$ (or $\left.\star_{f}\right)$. To end this note, we study briefly this problem.

Let $D$ be an integral domain and $\star$ a semistar operation on $D$. We say that $D$ is a quasi- $\star$-Dedekind domain (a $\mathrm{Q} \star \mathrm{DD})$ if $D$ is a $\star$-Noetherian $\mathrm{QP} \star \mathrm{MD}$. It is clear that a $\star$-Dedekind domain is a quasi- $\star$-Dedekind domain and that $D$ is a $\mathrm{Q} \star \mathrm{DD}$ if and only if $D$ is a $\mathrm{Q} \star_{f} \mathrm{DD}$. Moreover, it is easily seen that if $\star_{1} \leq \star_{2}$ are two semistar operations on $D$, if $D$ is a $\mathrm{Q} \star_{1} \mathrm{DD}$, then $D$ is a $\mathrm{Q} \star_{2} \mathrm{DD}$. Note also that obviously for a (semi)star operation $\star$ the notions of $\star \mathrm{DD}$ and $\mathrm{Q} \star \mathrm{DD}$ coincide.

Example 3.1. (1) An example of a $\mathrm{A} \star \mathrm{DD}$ which is not a $\star$-DD is given by the ring in Example 2.3 if one takes $D$ Noetherian. Indeed, in this case, $D$ is $\star$-Noetherian (since it is Noetherian) and we have seen that it is a $\mathrm{QP} \star \mathrm{MD}$, so it is a $\mathrm{Q} \star \mathrm{DD}$, but it is not a $\star-\mathrm{DD}$ since it is not a $\mathrm{P} \star \mathrm{MD}$. Note that this is also an example of a $\mathrm{Q} \star \mathrm{DD}$ which is not a $\mathrm{Q} \approx \mathrm{DD}$. 
(2) Similarly to the case of $\mathrm{QP} \star \mathrm{MD}$ (Example 2.6), it is not difficult to show that a domain with Dedekind integral closure (e.g., a one dimensional Noetherian domain) is always a quasi-b-Dedekind domain and it is never a $b$ Dedekind domain if it is not Dedekind (again because $\tilde{b}=d$ and so $b$-Dedekind is equivalent to Dedekind).

Note that the notion of $\mathrm{Q} \star \mathrm{DD}$ does not ascend to overrings (this depends on the fact that the notion of $\star$-Noetherian domain does not ascend), as the next example shows:

Example 3.2. Let $D$ be a Noetherian domain with a non-Noetherian valuation overring $V$, and $\iota$ the canonical embedding of $D$ in $V$. Let $\star:=\star\{V\}$. It is easy to see that $D$ is a quasi- $\star$-Dedekind domain. Since $\star_{\iota}=d_{V}$ is the identity semistar operation on $V$, we have that $V$ is not a quasi- $\star_{\iota}$-Dedekind domain, since it is not a Dedekind domain.

Proposition 3.3. Let $D$ be an integral domain, $T$ an overring of $D, \iota$ the canonical embedding of $D$ in $T$, $\star$ a semistar operation on $D$ and $*$ a semistar operation on $T$.

(1) If $T$ is a $Q * D D$, then $D$ is a $Q *^{\iota} D D$. (In particular, if $D^{\star}$ is $a \star_{\iota}-D D$, then $D$ is a $Q \star D D$.)

(2) If $\star$ is stable and $D$ is a $Q \star D D$, then $D^{\star}$ is a $Q \star D D$. (More precisely, $D^{\star}$ is a Krull domain and $\star=v\left(D^{\star}\right)$.)

Proof. (1) It follows from Proposition 2.10 and [2, Lemma 3.1].

(2) $D^{\star}$ is a $\mathrm{QP} \star_{\iota} \mathrm{MD}$ by Proposition 2.10. Moreover, since $\star$ is stable, $D^{\star}$ is $\star_{\iota}$-Noetherian $([2$, Proposition 3.4$])$. The parenthetical statement is a consequence of $\left[2\right.$, Proposition 4.5], since $\star_{\iota}$ is a (semi)star operation on $D^{\star}$.

Proposition 3.4. Let $D$ be an integral domain and $\star$ a semistar operation on $D$. The following are equivalent:

(i) $D$ is $a$ $Q \star D D$.

(ii) Each nonzero ideal of $D$ is quasi- $\star_{f}$-invertible.

Proof. (i) $\Rightarrow$ (ii) Let $I$ be a nonzero ideal of $D$. Since $D$ is $\star$-Noetherian, $I^{\star}=J^{\star} f$ for a finitely generated ideal $J \subseteq I$ of $D([2$, Lemma 3.3]). Since $D$ is a $\mathrm{QP} \star \mathrm{MD}$, $J$ is quasi- $\star_{f}$-invertible. So, $D^{\star}=\left(J\left(D^{\star}: J\right)\right)^{\star_{f}}=\left(J^{\star_{f}}\left(D^{\star}: J^{\star_{f}}\right)\right)^{\star_{f}}=$ $\left(I^{\star}\left(D^{\star}: I^{\star}\right)\right)^{\star}=\left(I\left(D^{\star}: I\right)\right)^{\star}$.

(ii) $\Rightarrow$ (i) It follows from [7, Lemma 2.14] and [2, Lemma 3.3].

Corollary 3.5. Let $D$ be an integral domain and $\star$ a semistar operation on $D$. The following are equivalent:

(i) $D$ is $a \star-D D$.

(ii) $D$ is a quasi- $\tilde{\star}-D D$.

Proof. It is immediate from Proposition 3.4 since a $\star$-DD is a domain in which each ideal is quasi- $\tilde{\star}$-invertible [2, Proposition 4.1]. 
Remark 3.6. A $\star$-Dedekind domain is characterized by the fact that each nonzero ideal is quasi- $\tilde{\star}$-invertible, and not $\star_{f}$-invertible, as one would expect considering that $\mathrm{P} \star \mathrm{MDs}$ are defined using $\star_{f}$-invertibility. From this point of view, things work well for $\mathrm{QP} \star \mathrm{MD}$ 's and $\mathrm{Q} \star \mathrm{DD}$ 's, in fact, $\mathrm{QP} \star \mathrm{MD}$ 's, which are defined using quasi- $\star_{f}$-invertibility, give rise to $\mathrm{Q} \star \mathrm{DD}$ 's, which are characterized using quasi- $\star_{f}$-invertibility, as shown in Proposition 3.4. Note that this recovers also the case of a $\mathrm{P} \star \mathrm{MD}$ : indeed, we have shown in Proposition 2.2 that a $\mathrm{P} \star \mathrm{MD}$ is a domain in which all finitely generated ideals are quasi- $-\tilde{\star}-$ invertible (i.e., a $\mathrm{QP} \approx \mathrm{MD}$ ), and so it is natural that they give rise to the $\star-\mathrm{DD}$

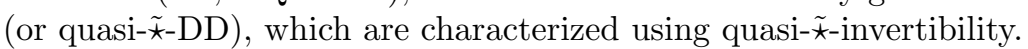

To finish, we recall that Dedekind domains can be characterized as the Noetherian, integrally closed, one-dimensional domains. The analogous characterization for semistar Dedekind domains holds, using the notion of quasi- $\tilde{\star}$-integral closure and $\tilde{\star}$-dimension (see [2, Theorem 4.15]). (Recall that the $\star$-dimension of $D$ is the supremum of the lengths of the chains of quasi- $\star$-prime ideals.)

It is easy to see that a $\mathrm{Q} \star \mathrm{DD}$ is quasi- $\star$-integrally closed (a domain $D$ is quasi- $\star$-integrally closed if $\left(I^{\star}: I^{\star}\right) \subseteq D^{\star}$ for all finitely generated nonzero ideals $I$ of $D)$. This fact is an easy consequence of [2, Lemma 4.13(1)]. Moreover, by definition, $\mathrm{Q} \star \mathrm{DD}$ is $\star$-Noetherian.

With the next example, we show that a $\mathrm{Q} \star \mathrm{DD}$ can have $\star$-dimension higher than one, even when $\star$ is a semistar operation of finite type. It follows that the most classical characterization of Dedekind domains cannot be transferred to the semistar case, when $\star \neq \tilde{\star}$, that is when $D$ is a $\mathrm{Q} \star \mathrm{DD}$ that is not a $\star$-DD.

Example 3.7. Let $D$ be a Noetherian 2-dimensional domain. Let $P_{1} \subsetneq P_{2}$ be two primes of $D$. By [9, Corollary 19.7], there exists a valuation overring $V$ of $D$ with prime ideals $M_{1} \subsetneq M_{2}$ contracting respectively to $P_{1}$ and $P_{2}$. Let $\star:=\star\{V\}$ be the finite type semistar operation given by the extension to the overring $V$. Clearly $D$ is a $\mathrm{Q} \star \mathrm{DD}$ because it is $\star$-Noetherian and a $\mathrm{QP} \star \mathrm{MD}$ (since $D^{\star}=V$ is Prüfer). But it is easily seen that $P_{1}$ and $P_{2}$ are quasi- $\star$-ideals. Since $P_{1} \subsetneq P_{2}$ we have $\star-\operatorname{dim}(D)=2$.

\section{References}

[1] J. T. Arnold and J. W. Brewer, Kronecker function rings and flat $D[X]$-modules, Proc. Amer. Math. Soc. 27 (1971), 483-485.

[2] S. El Baghdadi, M. Fontana, and G. Picozza, Semistar Dedekind domains, J. Pure Appl. Algebra 193 (2004), no. 1-3, 27-60.

[3] S. El Baghdadi and M. Fontana, Semistar linkedness and flatness, Prüfer semistar multiplication domains, Comm. Algebra 32 (2004), no. 3, 1101-1126.

[4] M. Fontana and J. A. Huckaba, Localizing systems and semistar operations, NonNoetherian commutative ring theory, 169-197, Math. Appl., 520, Kluwer Acad. Publ., Dordrecht, 2000.

[5] M. Fontana, P. Jara, and E. Santos, Prüfer $\star$-multiplication domains and semistar operations, J. Algebra Appl. 2 (2003), no. 1, 21-50.

[6] M. Fontana and K. A. Loper, Nagata rings, Kronecker function rings, and related semistar operations, Comm. Algebra 31 (2003), no. 10, 4775-4805. 
[7] M. Fontana and G. Picozza, Semistar invertibility on integral domains, Algebra Colloq. 12 (2005), no. 4, 645-664.

[8] R. Gilmer, An embedding theorem for HCF-rings, Proc. Cambridge Philos. Soc. 68 (1970), 583-587.

[9] Multiplicative Ideal Theory, Corrected reprint of the 1972 edition. Queen's Papers in Pure and Applied Mathematics, 90. Queen's University, Kingston, ON, 1992.

[10] M. Griffin, Some results on v-multiplication rings, Canad. J. Math. 19 (1967), 710-722.

[11] J. R. Hedstrom and E. G. Houston, Pseudo-valuation domains, Pacific J. Math. 75 (1978), no. 1, 137-147.

[12] E. G. Houston, S. B. Malik, and J. L. Mott, Characterizations of *-multiplication domains, Canad. Math. Bull. 27 (1984), no. 1, 48-52.

[13] B. G. Kang, Prüfer v-multiplication domains and the ring $R[X]_{N_{v}}$, J. Algebra 123 (1989), no. $1,151-170$.

[14] A. Okabe and R. Matsuda, Semistar-operations on integral domains, Math. J. Toyama Univ. 17 (1994), 1-21.

[15] G. Picozza, Star operations on overrings and semistar operations, Comm. Algebra 33 (2005), no. 6, 2051-2073.

[16] - A note on semistar Noetherian domains, Houston J. Math. 33 (2007), no. 2, $415-432$.

[17] G. Picozza and F. Tartarone, When the semistar operation $\tilde{\star}$ is the identity, Comm. Algebra 36 (2008), no. 5, 1954-1975.

[18] J. Querré, Idéaux divisoriels d'un anneau de polynômes, J. Algebra 64 (1980), no. 1, $270-284$.

[19] F. Wang and R. L. McCasland, On w-modules over strong Mori domains, Comm. Algebra 25 (1997), no. 4, 1285-1306.

[20] M. Zafrullah, Some polynomial characterizations of Prüfer v-multiplication domains, J. Pure Appl. Algebra 32 (1984), no. 2, 231-237.

Université Paul Cézanne Aix-Marseille III LATP, Faculté des Sciences et Techniques 13397 Marseille Cedex 20, France

E-mail address: giampaolo.picozza@univ-cezanne.fr 